Conclusion Findings support the biological hypothesis of the importance of mode, timing of birth and breastfeeding in development of gut microbiota and immune system in early life. Spontaneous vaginal birth at $39+$ weeks gestation with any exposure to breastmilk at birth minimises the risk of AGE hospital admission in early childhood.

\section{0-172 INFANT FEEDING AND ANTI-TISSUE TRANSGLUTAMINASE ANTIBODY LEVELS IN CHILDREN WITH SUBCLINICAL CELIAC DISEASE: THE GENERATION R STUDY}

${ }^{1}$ IIM Tromp, ${ }^{1}$ MAE Jansen, ${ }^{2} \mathrm{JC}$ Kiefte-de Jong, ${ }^{3} \mathrm{VWV}$ Jaddoe, ${ }^{2} \mathrm{~A}$ Hofman, ${ }^{4} \mathrm{JC}$ Escher, ${ }^{5} \mathrm{H}$ Hooijkaas, ${ }^{6}$ HA Moll. ${ }^{1}$ Pediatrics/The Generation R Study Group, Erasmus University Medical Center, Rotterdam, Netherlands; 'Epidemiology, Erasmus University Medical Center, Rotterdam, Netherlands; ${ }^{3}$ The Generation R Study Group/Pediatrics/ Epidemiology, Erasmus University Medical Center, Rotterdam, Netherlands; ${ }^{4}$ Pediatric Gastroenterology, Erasmus University Medical Center, Rotterdam, Netherlands; ${ }^{5}$ Immunology, Erasmus University Medical Center, Rotterdam, Netherlands; ${ }^{6}$ Pediatrics, Erasmus University Medical Center, Rotterdam, Netherlands

\subsection{6/archdischild-2014-307384.241}

Objective To examine whether the timing of gluten introduction and breastfeeding duration are associated with subclinical celiac disease in children at the age of 6 years.

Methods This study was embedded in the Generation R study, a population-based prospective cohort study. Participants included 1679 Dutch children positive for HLA-DQ2/DQ8. Data on the timing of gluten introduction ( $<6$ months vs. $\geq 6$ months) and duration of breastfeeding ( $<6$ months vs. $\geq 6$ months) were obtained by questionnaire. Serum samples were analysed for anti-tissuetransglutaminase (tTG) levels at age 6 years. Anti-tTG levels were categorised into negative $(\leq 7 \mathrm{U} / \mathrm{ml})$ and positive $(>7$ $\mathrm{U} / \mathrm{ml}$ ) levels. Positive anti-tTG levels were further categorised based on the $\geq 10$ times upper limit of normal (ULN) levels of the test kit $(>7-70 \mathrm{U} / \mathrm{ml}$ and $\geq 70 \mathrm{U} / \mathrm{ml})$. Multivariable logistic regression analyses were performed.

Results Positive anti-tTG levels were found in 43 children of which 26 children had levels above the 10 times ULN ( $\geq 70$ IU/ $\mathrm{ml})$. The introduction of gluten from the age of 6 months onwards and breastfeeding for 6 months or longer were not significantly associated with positive anti-tTGlevels. In addition, the timing of gluten introduction and duration of breastfeeding were not significantly associated with positive anti-tTG levelsbelow and above the 10 times ULN.

Conclusions Delayed introduction of gluten beyond the age of 6 months does not increase the risk of subclinical CD. Also, breastfeeding for 6 months or longer does not decrease the risk of subclinical CD in children at 6 years of age.

\section{0-173 EVALUATING THE POTENTIAL ROLE OF SMALL INTESTINE CONTRAST ULTRASONOGRAPHY IN PAEDIATRIC CROHN'S DISEASE: 5 YEAR EXPERIENCE IN A SINGLE CENTRE}

${ }^{1}$ A Hakim, ${ }^{1} S$ Kumar, ${ }^{2} \mathrm{C}$ Alexakis, ${ }^{3} \mathrm{D}$ Tzias, ${ }^{3} \mathrm{~J}$ Pilcher, ${ }^{2} \mathrm{R}$ Pollok. 'Medical School, St George's Hospital, London, UK; '2Department of Gastroenterology, St George's Hospital, London, UK; ${ }^{3}$ Department of Radiology, St George's Hospital, London, UK

\subsection{6/archdischild-2014-307384.242}

Background and aims Small intestine contrast ultrasonography (SICUS) is an emerging, non-invasive technique which accurately assesses small bowel lesions associated with Crohn's disease (CD) in adult patients, without exposure to medical radiation. We report our 5 year experience in a paediatric cohort.

Methods Patients with suspected or established CD who underwent SICUS were identified and radiological findings collated. SICUS was compared to conventional transabdominal ultrasound (TUS), ileocolonoscopy and magnetic resonance enterography (MRE). Accuracy and agreement of SICUS in detecting small bowel lesions and CD-related complications was assessed using kappa $(\kappa)$ coefficient statistics.

Results 93 patients (median age 16 years, range 2-20, 49 male) underwent SICUS; 58 had suspected and 35 established CD. In suspected CD, sensitivity and specificity of SICUS in detecting CD small bowel lesions were $81.82 \%$ and $100 \%$ and TUS $85.71 \%$ and $87.50 \%$, respectively. In established CD, sensitivity and specificity of SICUS were $83.33 \%$ and $100 \%$ and TUS $80.00 \%$ and $100 \%$, respectively. Agreement with ileocolonoscopy was fair for the presence of lesions (SICUS, $\kappa=0.38$, TUS, $\kappa=0.31$ ). Agreement between SICUS and ileocolonoscopy was good for detecting strictures $(\kappa=0.66)$ with a sensitivity of $100 \%$ and specificity of $97.62 \%$. Comparing SICUS and TUS with MRE, agreement for the presence of lesions was $\kappa=0.63$ and 0.53 , respectively. Agreement between SICUS and MRE was good for detecting strictures $(\kappa=0.77)$ and fair for assessing dilatation $(\kappa=0.45)$.

Conclusions SICUS offers a promising radiation-free, low cost alternative for diagnosing and monitoring paediatric CD small bowel complications. Its wider use should be adopted.

\section{0-174 THE COMBINATION OF SCGOS/LCFOS WITH FERMENTED INFANT FORMULA REDUCES THE INCIDENCE OF COLIC IN 4 WEEK OLD INFANTS}

${ }^{1} \mathrm{Y}$ Vandenplas, ${ }^{2} \mathrm{~T}$ Ludwig, ${ }^{2} \mathrm{H}$ Bouritius, ${ }^{3} \mathrm{~J}$ Hourihane, ${ }^{4} \mathrm{~F}$ Huet. ${ }^{1}$ Department of Pediatrics, UZ Brussels Free University of Brussels, Brussels, Belgium; ${ }^{2}$ Nutricia Research, Utrecht, Netherlands; ${ }^{3}$ Paediatrics and Child Health, University College Cork, Cork, Ireland; ${ }^{4}$ Pédiatrie, Hôpital d'Enfants, Dijon, France

\subsection{6/archdischild-2014-307384.243}

Background and aims The effects on gastrointestinal (GI) tolerance and prevalence of colics (secondary outcome parameters) of a novel infant formula (IF) were explored in a randomised, controlled, double-blind, multicenter intervention study on growth, safety and GI tolerance. The novel IF combined the fermented IF Lactofidus ${ }^{\mathrm{TM}}(\mathrm{LF})$ with short-chain galacto-oligosaccharides and long-chain fructo-oligosaccharides (scGOS/lcFOS, ratio 9:1, 0.8 $\mathrm{g} / 100 \mathrm{ml}$ ).

Methods 432 healthy, term infants aged 0-28 days were randomised after parent's autonomous decision to discontinue breastfeeding. IF with scGOS/lcFOS and 50\%LF (LF50+), IF with scGOS/lcFOS and 15\%LF (LF15+), and as controls IF with $50 \%$ LF (LF50), or IF with scGOS/lcFOS (IF+) were tested. Parents completed standardised 7-day diaries with daily entries on GI symptoms and crying in monthly intervals until 17 weeks of age. Colic was defined by adapted Rome III criteria.

Results Growth and safety outcomes were within the normal ranges. Based on low mean GI symptom-scores, the newly-developed IFs were well tolerated. The incidence of colic was highest $(16.1 \%)$ at the 4 week visit and in line with literature (i.e. $20.5 \%$ at 4 weeks of age [Iacono et al., 2005]). The incidence of colic was significantly lower with LF50+ (8\%) compared to IF+ $(20 \%)(\mathrm{p}=0.034$; chi-square test $)$, and LF50 $(20 \%)(\mathrm{p}=0.036)$ at the 4 week visit. Colic was found to be associated with 